\title{
On the solutions of L-fuzzy relational equations with sub-triangle composition and its relation with the L-Fuzzy Concept Theory
}

\author{
C.Alcalde ${ }^{1}$ A. Burusco, R. Fuentes-González ${ }^{2}$ \\ ${ }^{1}$ Escuela Universitaria Politécnica. Departamento de Matemática Aplicada. \\ UPV/EHU. Plaza de Europa 1, 20018 San Sebastián, Spain. \\ Email: c.alcalde@ehu.es \\ ${ }^{2}$ Departamento de Automática y Computación. Universidad Pública de Navarra. \\ Campus de Arrosadía, 31006 Pamplona, Spain. \\ Email: \{burusco,rfuentes\}@unavarra.es
}

\begin{abstract}
Given a Brouwerian complete lattice $(L, \leq)$ and two referential sets $K$ and $E$, and using a fuzzy relation $R \in L^{E \times E}$ which is reflexive and symmetric, certain fuzzy relations $\widehat{H} \in L^{K \times E}$ are characterized as solutions of $X \triangleleft R=X$. These solutions $\widehat{H}$ can be determinated by means of the L-fuzzy concepts associated with the K-labeled L-fuzzy context $(L, K, E, E, R)$.
\end{abstract}

Keywords: Fuzzy relational equations, $L$-fuzzy tolerances, Formal Concept Analysis, $L$-fuzzy Concept Theory.

\section{Introduction}

We start the introduction by recalling some aspects about $L$-fuzzy tolerances and the fundamental definitions and results about the $L$-fuzzy Concept Theory. Next, these two theories are related when we try to find the solutions of the equation $X \triangleleft R=X$ associated with a fuzzy tolerance $R \in L^{E \times E}$.

\section{1. $L$-fuzzy tolerances}

Let $(L, \leq, \vee, \wedge)$ be a complete Brouwerian lattice [7], i.e., such that

$$
\alpha \wedge \sup M=\sup (\alpha \wedge M), \quad \forall \alpha \in L, \quad \forall M \subseteq L .
$$

As a generalization of the classical tolerance spaces [14], we analyze in this work the $L$-tolerance spaces $(L, K \times E, R)$ in which $K$ and $E$ are two ordinary non empty referential sets, (that can be coincident: $K=E$ ), $K \times E$ is the usual product of ordinary sets and where $R \in L^{E \times E}$ is an $L$-fuzzy tolerance relation on $E$ ([23],[13]), also denoted $L$ fuzzy compatibility [19]. That is, $R$ is an application $R: E \times E \rightarrow L$ such that

$$
\begin{gathered}
R(x, x)=1, \forall x \in E \text { and } \\
R(x, y)=R(y, x), \forall(x, y) \in E \times E .
\end{gathered}
$$

In the following, the expression $R^{o p}$ represents the opposite relation of $R$, that is, $R^{o p}(x, y)=R(y, x)$, $\forall(x, y) \in E \times E$.

For every $S \in L^{K \times E}$ there is an associated family $\left(S_{w}\right)_{w \in K}$ of $L$-fuzzy subsets $S_{w} \in L^{E}$ such that $S_{w}(x)=S(w, x), \forall w \in K, \forall x \in E$.

Moreover, the elements that we will use in this work are the following:

- A left-continuous $t$-norm $\mathrm{T}$ on $L$, that is,

$$
\alpha \top \sup M=\sup (\alpha \top M), \forall \alpha \in L, \forall M \subseteq L .
$$

- The residuated implication $\rightsquigarrow$ associated with this t-norm:

$$
\alpha \rightsquigarrow \beta=\sup \{\gamma / \alpha \mathrm{T} \gamma \leq \beta\}, \forall(a, \beta) \in L^{2} .
$$

- Two non empty ordinary referential sets $K$ and E.

- The operator $\triangleleft: L^{K \times E} \times L^{E \times E} \longrightarrow L^{K \times E}$ associated with the implication $\rightsquigarrow$, defined $\forall S \in L^{K \times E}, \forall R \in L^{E \times E}$ and $\forall(w, y) \in K \times E$ as:

$$
(S \triangleleft R)(w, y)=\inf \{S(w, x) \rightsquigarrow R(x, y) / x \in E\}
$$

and that is said to be a $\rightsquigarrow-$ triangle.

- The operator $\widetilde{\times}: L^{E} \times L^{E} \longrightarrow L^{E \times E}$ associated with the $t$-norm $\mathrm{T}$, defined as:

$$
\begin{aligned}
& (A \widetilde{\times} B)(x, y)=A(x) \text { т } B(y), \\
& \forall(x, y) \in E \times E, \forall A, B \in L^{E}
\end{aligned}
$$

that is said to be a T- $\widetilde{\text { product. }}$.

Notice that as $L$ is a Brouwerian lattice, then at least a left-continuous $t$-norm exists.

Next, we define an extension of this T-product operator to the $L$-fuzzy relations that will be used in the next section. 
Definition 1 The T-product operator defined on the set of the $L$-fuzzy relations $\widetilde{\times}: L^{K \times E} \times$ $L^{K \times E} \longrightarrow L^{E \times E}$ is given by:

$$
S \widetilde{\times} Q=\bigvee_{w \in K}\left(S_{w} \widetilde{\times} Q_{w}\right)=S^{o p} \circ_{\mathrm{\top}} Q, \forall S, Q \in L^{K \times E}
$$

where ${ }^{\circ}$ is the usual composition of relations associated to the t-norm $\mathrm{T}$ on $L$, that is, $\forall(x, y) \in E \times E$ :

$$
(S \widetilde{\times} Q)(x, y)=\sup \{S(w, x) \text { Т } Q(w, y) / w \in K\}
$$

\section{2. $L$-fuzzy Concept Analysis}

The Formal Concept Analysis developed by Wille [26] tries to extract some information from a binary table that represents a Formal context $(E, F, R)$ with $E$ and $F$ two finite sets (of objects and attributes, respectively) and $R \subseteq E \times F$ the incidence relation.

This information is obtained by means of the formal concepts which are pairs $(A, B)$ with $A \subseteq E$, $B \subseteq F$ where $A$ is the extension and $B$ the intension of the formal concept. These pairs verify that $A^{*}=B$ and $B^{*}=A$ where $*$ is the derivation operator which associates to each object set $A$ the set $B$ of the attributes related to $A$, and viceversa:

$$
\begin{aligned}
& A^{*}=\{y / x R y \forall x \in A\} \\
& B^{*}=\{x / x R y \forall y \in B\}
\end{aligned}
$$

These formal concepts represent a group of objects $A$ that share some attributes $B$.

The set of the formal concepts is a complete lattice and is said to be the Formal concept lattice $(\mathcal{L}(E, F, R), \leq)([26],[16])$.

The order relation $\leq$ of the lattice $(\mathcal{L}(E, F, R), \leq)$ is given by:

$$
\begin{aligned}
& (A, B) \leq(C, D) \Longleftrightarrow A \subseteq C \\
& \text { (or, equivalently, } B \supseteq D) .
\end{aligned}
$$

There exist in the literature extensions of the formal concept theory to $L$-fuzzy subsets, ([9], [10], [12], [4], [5], [6], [20], [21], [2], [15], [22]). In these extensions, the incidence relation $R$ of the context $(L, E, F, R)$ is an $L$-fuzzy relation $R \in L^{E \times F}$. The concepts are pairs of $L$-fuzzy subsets $(A, B) \in L^{E} \times L^{F}$ obtained from certain structures on the lattice $L$.

For example, in [12] an implication operator $\rightsquigarrow$ : $L^{2} \rightarrow L$ is used to define the derivation operators ${ }_{1}: L^{E} \rightarrow L^{F}$ and ${ }_{2}: L^{F} \rightarrow L^{E}$ such that

$$
\begin{aligned}
& A_{1}(y)=\inf \{A(x) \rightsquigarrow R(x, y) / x \in E\}, \\
& B_{2}(x)=\inf \{B(y) \rightsquigarrow R(x, y) / y \in F\}, \\
& \quad \forall A \in L^{E}, \forall y \in F, \forall B \in L^{F}, \forall x \in E
\end{aligned}
$$

An $L$-fuzzy concept of the context $(L, E, F, R)$ associated with these operators, is a pair $(A, B) \in$ $L^{E} \times L^{F}$ such that $A_{1}=B$ and $B_{2}=A$.

The set of these $L$-fuzzy concepts $(A, B)$, that is represented by $\mathcal{L}(L, E, F, R)$, is a complete lattice with the order $\preccurlyeq$ defined by:

$$
\begin{aligned}
& (A, B) \preccurlyeq(C, D) \Longleftrightarrow A \leq C \\
& \text { (or, equivalently, } B \geq D \text { ), }
\end{aligned}
$$

where $\leq$ is the usual $L$-fuzzy inclusion.

The defined $L$-fuzzy subsets $A_{1}$ and $B_{2}$ are said to be the fuzzy extension and the fuzzy intension respectively, and can be expressed by means of the operator $\triangleleft$ associated with the implication operator, that is:

$$
A_{1}=A \triangleleft R, \quad B_{2}=B \triangleleft R^{o p},
$$

where $R^{o p} \in L^{F \times E}$ is the opposite relation of $R$ and $\triangleleft$ is a particular case of the definition given in the previous section.

On the other hand, we want to indicate that the referential sets $E$ and $F$ can be coincident. Some properties of the $L$-fuzzy concepts in contexts as $(L, E, E, R)$ can be seen in [1], in which we studied different types of relations $R \in L^{E \times E}$ (reflexive, symmetric, etc).

This $L$-fuzzy concept theory has been extended in [11] to some more general $L$-fuzzy contexts called K-labeled, $(L, K, E, F, R)$, where a new set $K$ is introduced. In this case, $E$ and $F$ are two ordinary referential sets of objects and attributes respectively and $R \in L^{E \times F}$ an $L$ fuzzy incidence relation. The set of labels $K$ is used to represent some important aspects of the study and is related to the object and attribute sets.

For instance, it is studied in [11] the situation where an academy offers some courses ( represented by $E$ ) for unemployed who want to train for a job $(F)$. By means of the use of L-fuzzy concepts we give to these unemployed some recommendations about the courses and the jobs taking into account their different motivations represented by the set of labels $(K)$.

In this case, the derivation operators are defined using the relations in the analogous way:

$$
\begin{aligned}
S_{1}(w, y)= & (S \triangleleft R)(w, y)= \\
& =\inf \{S(w, x) \rightsquigarrow R(x, y) / x \in E\}, \\
& \forall S \in L^{K \times E}, \forall(w, y) \in K \times F, \\
Q_{2}(w, x)= & \left(Q \triangleleft R^{o p}\right)(w, x)= \\
& =\inf \{Q(w, y) \rightsquigarrow R(x, y) / y \in F\}, \\
& \forall Q \in L^{K \times F}, \forall(w, x) \in K \times E .
\end{aligned}
$$


and the $L$-fuzzy concepts are now the pairs of $L$-fuzzy relations $(S, Q)$ such that $S_{1}=Q$ and $Q_{2}=S$. The order is defined in the analogous way to the mentioned above.

Although the referential sets $E$ and $F$ of the $L$ fuzzy context and the lattice $L$ can have any cardinality, from the point of view of the applications, the interesting cases are those in which the cardinality is finite. This is the situation in the following example:

Example 1 Let $(L, K, E, F, R)$ be the $K$-labeled $L$ fuzzy context with $L=\{0,0.2,0.4,0.6,0.8,1\}, K=$ $\left\{k_{1}, k_{2}, k_{3}\right\}$ and coincident sets of objects and attributes, $E=F=\left\{x_{1}, x_{2}, x_{3}, x_{4}, x_{5}\right\}$. Let us suppose the following reflexive and symmetric relation $R \in L^{E \times E}$ :

\begin{tabular}{c|ccccc}
$R$ & $x_{1}$ & $x_{2}$ & $x_{3}$ & $x_{4}$ & $x_{5}$ \\
\hline$x_{1}$ & 1 & 1 & 0.2 & 0 & 0 \\
$x_{2}$ & 1 & 1 & 1 & 0.8 & 0 \\
$x_{3}$ & 0.2 & 1 & 1 & 1 & 0 \\
$x_{4}$ & 0 & 0.8 & 1 & 1 & 0.2 \\
$x_{5}$ & 0 & 0 & 0 & 0.2 & 1
\end{tabular}

We show here two K-labeled L-fuzzy concepts that have been calculated using as implication $\rightsquigarrow$ the Lukasiewicz implication operator:

$$
\begin{aligned}
& (S 1, Q 1)=\left(\begin{array}{c|ccccc} 
& x_{1} & x_{2} & x_{3} & x_{4} & x_{5} \\
\hline k_{1} & 0.2 & 1 & 0.2 & 0 & 0 \\
k_{2} & 0 & 0.8 & 1 & 0.8 & 0 \\
k_{3} & 1 & 1 & 0.2 & 0 & 0
\end{array}\right. \\
& (S 2, Q 2)=\left(\begin{array}{c|ccccc} 
& x_{1} & x_{2} & x_{3} & x_{4} & x_{5} \\
\hline k_{1} & 0 & 0 & 0 & 0.2 & 1 \\
k_{2} & 0.2 & 1 & 1 & 0.8 & 0 \\
k_{3} & 1 & 1 & 0.2 & 0 & 0
\end{array},\right.
\end{aligned}
$$

$\left.\begin{array}{c|ccccc} & x_{1} & x_{2} & x_{3} & x_{4} & x_{5} \\ \hline k_{1} & 1 & 1 & 1 & 0.8 & 0 \\ k_{2} & 0.2 & 1 & 1 & 1 & 0 \\ k_{3} & 1 & 1 & 0.2 & 0 & 0\end{array}\right)$

$\left.\begin{array}{c|ccccc} & x_{1} & x_{2} & x_{3} & x_{4} & x_{5} \\ \hline k_{1} & 0 & 0 & 0 & 0.2 & 1 \\ k_{2} & 0.2 & 1 & 1 & 0.8 & 0 \\ k_{3} & 1 & 1 & 0.2 & 0 & 0\end{array}\right)$

The meaning of these concepts will be explained in the next section.

Note that in the second case $S 2=Q 2$. These $K$ labeled $L$-fuzzy concepts that have coincident extension and intension will be of special interest.

\section{Solutions of the equation $X \triangleleft R=X$}

The study of the eigen fuzzy sets equations of the type $R \circ X=X$ was introduced by Sanchez[24], with applications to medical diagnosis. There are more references about these equations in the bibliography $([25,17])$.

In this paper, we are interested in solving the equation $X \triangleleft R=X$ which solutions can be interpreted as eigenvalues associated with the operator $\triangleleft$ instead of $\circ$. We prove that if $R$ is a fuzzy tolerance, then the set of eigenvalues is not a void set and we give an algorithm for their calculus related with the Fuzzy set theory.

Proposition 1 The following equivalence is fulfilled:

$$
(S \widetilde{\times} S) \leq R \Leftrightarrow S \leq(S \triangleleft R)
$$

Proof: Let us suppose that $S \widetilde{\times} Q \leq R$. Then, $S(w, x)$ † $Q(w, y) \leq R(x, y), \quad \forall x, y \in E, \forall w \in K$.

Let $w$ and $y$ be two elements of $E$, then, as the implication $\rightsquigarrow$ is the residuum ([8]) of the $t$-norm T, it is verified that:

$$
Q(w, y) \leq(S(w, x) \rightsquigarrow R(x, y)), \quad \forall x \in E,
$$

therefore

$Q(w, y) \leq \inf _{x \in E}(S(w, x) \rightsquigarrow R(x, y))=(S \triangleleft R)(w, y)$.

And this happens for every pair of elements $y$ and $w$, hence,

$$
Q \leq(S \triangleleft R) .
$$

To prove the inverse implication, let us suppose now that $Q \leq(S \triangleleft R)$, then:

$Q(w, y) \leq(S(w, x) \rightsquigarrow R(x, y)), \forall w \in K, \forall x, y \in E$,

and, consequently,

$$
S(w, x) \text { Т } Q(w, y) \leq R(x, y), \forall w \in K, \forall x, y \in E .
$$

That is:

$$
S \widetilde{\times} Q \leq R
$$

Now, we study the classes of $L$-fuzzy sets $\mathcal{S}_{R}$ and $\mathcal{H}_{R}$, characterized by the relation $R$ and the operator $\triangleleft$.

To do this, we consider the ordinary set $\mathcal{S}_{R} \subset$ $L^{K \times E}$ :

$$
\mathcal{S}_{R}=\left\{S \in L^{K \times E} / S \leq(S \triangleleft R)\right\}
$$

And the ordinary set $\mathcal{H}_{R} \subset \mathcal{S}_{R}$ :

$$
\mathcal{H}_{R}=\left\{H \in L^{K \times E} / H=(H \triangleleft R)\right\}
$$

$\mathcal{H}_{R}$ can be empty, but since $\varnothing \in \mathcal{S}_{R}, \mathcal{S}_{R} \neq \varnothing$. 
Proposition 2 It is true that:

1. If $R$ is an L-fuzzy tolerance, then $\mathcal{H}_{R}=$ $M A X I M A L\left(\mathcal{S}_{R}\right) \neq \varnothing$.

2. The subset $\mathcal{N H}_{R} \subset \mathcal{H}_{R}$ of the maximals of $\mathcal{S}_{R}$ defined by:

$$
\begin{aligned}
\mathcal{N H}_{R}= & \{\widehat{H} \quad /(\widehat{H}=(\widehat{H} \triangleleft R)) \\
& \left.\left(\widehat{H}_{k} \text { is normal, } \forall k \in K\right)\right\},
\end{aligned}
$$

where $\widehat{H}_{k} \in L^{E}$ is such that $\widehat{H}_{k}(x)=\widehat{H}(k, x)$, $\forall x \in E$, verifies that $\mathcal{N} \mathcal{H}_{R} \neq \varnothing$.

\section{Proof:}

1. As we know that $\mathcal{H}_{R} \subseteq M A X I M A L\left(\mathcal{S}_{R}\right)$ holds, then we have to prove the other inclusion:

Let $S \in M A X I M A L\left(\mathcal{S}_{R}\right)$, then $S \leq(S \triangleleft R)$. Let us suppose that $S<(S \triangleleft R)$. Then, there exists at least one $(w, t) \in K \times E$ such that $S(w, t)<(S \triangleleft R)(w, t)$.

Let be $S_{w t} \in L^{K \times E}$ defined by:

$$
S_{w t}(k, x)= \begin{cases}S(k, x) & \text { if }(k, x) \neq(w, t) \\ (S \triangleleft R)(w, t) & \text { if }(k, x)=(w, t)\end{cases}
$$

Then, it is verified that:

$$
S<S_{w t}=S \vee \underline{(w, t)}_{(S \triangleleft R)(w, t)},
$$

where

$$
\begin{aligned}
\underline{(w, t)}_{(S \triangleleft R)(w, t)}(k, x)= \\
\quad= \begin{cases}0 & \text { if }(k, x) \neq(w, t) \\
(S \triangleleft R)(w, t) & \text { if }(k, x)=(w, t)\end{cases}
\end{aligned}
$$

Thus,

$$
\begin{aligned}
S_{w t} \triangleleft R & =\left(S \vee \underline{(w, t)}_{(S \triangleleft R)(w, t)}\right) \triangleleft R= \\
& \left.=(S \triangleleft R) \wedge \underline{(\underline{(w, t)}}_{(S \triangleleft R)(w, t)} \triangleleft R\right) .
\end{aligned}
$$

We will prove now that $S_{w_{t}} \leq\left(S_{w t} \triangleleft R\right)$ :

- $\forall k, \neq w$, taking into account the definition of $\underline{(w, t)}$ and that

$$
0 \rightsquigarrow \beta=1, \forall \beta \in L,
$$

it is verified:

$$
\begin{aligned}
& \left(S_{w t} \triangleleft R\right)(k, x)= \\
& =(S \triangleleft R)(k, x) \wedge\left(\underline{(w, t)}_{(S \triangleleft R)(w, t)} \triangleleft R\right)(k, x) \\
& =(S \triangleleft R)(k, x) \wedge \\
& \inf \left\{\left(\underline{(w, t)}_{(S \triangleleft R)(w, t)}(k, z)\right) \rightsquigarrow R(z, x) / z \in E\right\}= \\
& =(S \triangleleft R)(k, x) \wedge 1= \\
& =(S \triangleleft R)(k, x) \geq S(k, x)=S_{w t}(k, x)
\end{aligned}
$$

- $\forall k=w$ and $x \neq t$, taking into account that:

$$
\alpha \leq(\alpha \rightsquigarrow \beta) \rightsquigarrow \beta
$$

proven in [4] and that $R(x, w)=R(w, x)$ holds, it follows:

$$
\begin{aligned}
& \left(S_{w t} \triangleleft R\right)(k, x)=\left(S_{w t} \triangleleft R\right)(w, x)= \\
& (S \triangleleft R)(w, x) \wedge\left(\underline{(w, t)}_{(S \triangleleft R)(w, t)} \triangleleft R\right)(w, x) \\
& =(S \triangleleft R)(w, x) \wedge((S \triangleleft R)(w, t) \rightsquigarrow R(t, x)) \\
& =(S \triangleleft R)(w, x) \wedge \\
& \quad\left(\inf _{z \in E}\{S(w, z) \rightsquigarrow R(z, t)\} \rightsquigarrow R(t, x)\right) \geq \\
& (S \triangleleft R)(w, x) \wedge \\
& \quad((S(w, x) \rightsquigarrow R(x, t)) \rightsquigarrow R(t, x))= \\
& =(S \triangleleft R)(w, x) \wedge \\
& \quad((S(w, x) \rightsquigarrow R(t, x)) \rightsquigarrow R(t, x)) \geq \\
& (S \triangleleft R)(w, x) \wedge S(w, x)= \\
& =S(w, x)=S_{w t}(w, x) .
\end{aligned}
$$

- $\forall(k, x)=(w, t)$, taking into account that $R(w, w)=1$ :

$$
\begin{aligned}
& \left(S_{w t} \triangleleft R\right)(k, x)=\left(S_{w t} \triangleleft R\right)(w, t)= \\
& \inf _{z \in E}\left\{S_{w t}(w, z) \rightsquigarrow R(z, t)\right\}= \\
& \inf _{z \neq t}\left\{S_{w t}(w, z) \rightsquigarrow R(z, t)\right\} \wedge\left(S_{w t}(w, t) \rightsquigarrow R(t, t)\right) \\
& =\inf _{z \neq t}\{S(w, z) \rightsquigarrow R(z, t)\} \wedge 1 \geq \\
& \inf _{z \in E}\{S(w, z) \rightsquigarrow R(z, t)\}= \\
& (S \triangleleft R)(w, t)=S_{w t}(w, t)
\end{aligned}
$$

Then $S<S_{w t} \leq\left(S_{w t} \triangleleft R\right)$, that is, $S<S_{w t} \in \mathcal{S}_{R}$, but this is wrong since $S$ is a maximal element of $\mathcal{S}_{R}$. Consequently, $S=S \triangleleft R$, and $S \in \mathcal{H}_{R}$ is proven.

2. We consider the set of maps $E^{K}$ and we associate $\varphi \in E^{K}$ with the binary relation $Q_{(\varphi)} \in$ $L^{K \times E}$ defined by

$$
Q_{(\varphi)}(k, x)= \begin{cases}1 & \text { if } x=\varphi(k) \\ 0 & \text { in other case }\end{cases}
$$

We will prove that $Q_{(\varphi)} \in \mathcal{S}_{R}$, that is, $Q_{(\varphi)} \leq Q_{(\varphi)} \triangleleft R, \forall \varphi \in E^{K}$ :

We only have to verify that, $\forall k \in K,\left(Q_{(\varphi)} \triangleleft R\right)(k, \varphi(k))=1:$

$$
\begin{aligned}
& \left(Q_{(\varphi)} \triangleleft R\right)(k, \varphi(k))= \\
& =\inf \left\{Q_{(\varphi)}(k, x) \rightsquigarrow R(x, \varphi(k)) / x \in E\right\}= \\
& 1 \rightsquigarrow R(\varphi(k), \varphi(k))=1 \rightsquigarrow 1=1 .
\end{aligned}
$$

Then, there is a maximal element $\widehat{H} \in$ $M A X I M A L\left(\mathcal{S}_{R}\right)$ such that $Q_{(\varphi)} \leq \widehat{H}$ and evidently $\widehat{H}(k, \varphi(k))=1$. 
Therefore, for every $k \in K$ exists $x=\varphi(k) \in$ $E$ such that $\widehat{H}(k, x)=1$. Consequently $\widehat{H} \in$ $\mathcal{N} \mathcal{H}_{R}$.

At this point, from Proposition 2, we are on conditions to find the solutions of the equation $X \triangleleft R=X$ when $R$ is reflexive and simetrical.

Corollary 1 If $R$ is an L-fuzzy tolerance, the equation $X \triangleleft R=X$ has solutions in $L^{K \times E}$, and the set of solutions is the set of maximals $L$-fuzzy relations $H \in L^{K \times E}$. Consequently, every element $\widehat{H} \in \mathcal{N H}_{R}$ is a solution of this equation.

Moreover, from Proposition 1 and Proposition 2, these solutions verify that $H \tilde{\times} H \leq R$ that is related to the blocks in the context of the crisp tolerance relations.

On the other hand, by the definition of L-fuzzy concepts in the L-fuzzy context $(L, K, E, E, R)$, these solutions are the extension (or intension) of some special L-Fuzzy concepts where the extension and intension are coincident and formed by normal L-fuzzy sets.

Example 2 If we come back to Example 1, we can consider the $k$-labeled L-Fuzzy Context $(L, K, E, E, R)$ where $K$ is the set of tasks that a group of workers $(E)$ has to do and the tolerance relation $R$, the opinion of the person in charge about the relationship between the different workers.

Then, some solutions of the equation are the following ones:

\begin{tabular}{rl|ccccc} 
& $x_{1}$ & $x_{2}$ & $x_{3}$ & $x_{4}$ & $x_{5}$ \\
\hline$k_{1}$ & 0 & 0 & 0 & 0.2 & 1 \\
$k_{2}$ & 0.2 & 1 & 1 & 0.8 & 0 \\
$k_{3}$ & 1 & 1 & 0.2 & 0 & 0 \\
$H 2=$ & & $x_{1}$ & $x_{2}$ & $x_{3}$ & $x_{4}$ & $x_{5}$ \\
\hline$k_{1}$ & 0.2 & 1 & 1 & 0.8 & 0 \\
$k_{2}$ & 0 & 0.8 & 1 & 1 & 0 \\
$k_{3}$ & 1 & 1 & 0.2 & 0 & 0
\end{tabular}

since they are the extensions (or intensions) of some $K$-labeled L-fuzzy concepts where both elements of the pair are equals, and then verify the equation $H \triangleleft R=H$.

These solutions can be understood as the different ways of doing the tasks by the workers, taking into account the relationship among them (if the relation of two workers is bad, it is better no to put both in the same task).

Moreover, these solutions (that are the set of maximals $L$-fuzzy relations $H \in L^{K \times E}$ such that $H \widetilde{\times}$ $H \leq R)$ can be interpreted as follows:

$$
\forall k \in K, H(k, x) \text { Т } H(k, y) \leq R(x, y)
$$

That is: if $x$ and $y$ are involved in the same task $k$, then they must have a certain compatibility degree (expressed in relation $R$ ).

\section{Conclusions and Future work}

In this paper, we have showed a relationship between the solutions of the equation $X \triangleleft R=X$ when $R$ is a fuzzy tolerance and the L-fuzzy concepts of certain L-fuzzy context.

In future works, we will study the conection between these solutions and the blocks [3] in the context of the crisp tolerance relations.

\section{Acknowledgment}

This work has been partially supported by the Research Group "Intelligent Systems and Energy (SI+E)" of the Basque Government, under Grant IT519-10 and by the Research Project of the Government of Navarra (Resolution 2031 of 2008).

\section{References}

[1] C. Alcalde, A. Burusco, R. Fuentes-González, Contextos con conjuntos de objetos y atributos iguales, in: Proc. of ESTYLF08, pages 85-89, Mieres - Langreo, 2008.

[2] C. Alcalde, A. Burusco, R. Fuentes-González, I. Zubia, Treatment of L-fuzzy contexts with absent values, Information Sciences 179(1-2):1-15, 2009.

[3] W. Bartol, J. Miró, K. Pióro, F.Rosselló, On the coverings by tolerance classes, Information Sciences 166:193-211, 2004.

[4] R. Bělohlávek, Fuzzy relational Systems, IFSR International Series on Systems Science and Engineering 20, 2002.

[5] R. Bělohlávek, T. Funioková, Similarity and Fuzzy Tolerance Spaces, J. Logic Computation 14 (6):828-855, 2004.

[6] R. Bělohlávek, Fuzzy Galois connections and fuzzy concept lattices: from binary relations to conceptual structures, in: V. Novak, I. Perfileva (Eds.), Discovering the World with Fuzzy Logic, pages 462-494, Physica-Verlag, 2000.

[7] G. Birkhoff, Lattice Theory, American Mathematical Society, Providence, Rhode Island, 1940. Third Edition, 1979.

[8] T.S. Blyth, M.F. Janowitz, Residuation Theory, Pergamon Press, 1972.

[9] A. Burusco, R. Fuentes-González, The Study of the L-fuzzy Concept Lattice, Mathware and Soft Computing 1 (3):209-218, 1994.

[10] A. Burusco, R. Fuentes-González, Construction of the L-fuzzy Concept Lattice, Fuzzy Sets and Systems 97 (1):109-114, 1998.

[11] A. Burusco, R. Fuentes-González, Formal Concept Analysis in E-contexts, in: International 
Conference on Conceptual Knowledge Processing, Darmstadt, 1996.

[12] A. Burusco, R. Fuentes-González, Concept lattices defined from implication operators, Fuzzy Sets and Systems 114 (1):431-436, 2000.

[13] M. Das, M.K. Chakraborty, T.K. Ghoshal, Fuzzy tolerance relation, fuzzy tolerance space and basis, Fuzzy Sets and Systems 97:361-369, 1998.

[14] P. Doherty, W. Lukaszewicz, A. Szalas, Tolerance spaces and approximative representational structures, Lect. Notes in Computer Sciences 2821, pages 475-489, 2003.

[15] Y. Djouadi, D. Dubois, H. Prade, On the possible meanings of degrees when making formal concept analysis fuzzy, in: EUROFUSE workshop. Preference Modelling and Decision Analysis, Pamplona, pages 253-258, 2009.

[16] B. Ganter, R. Wille, Formal Concept Analysis, Springer Verlag, 1999.

[17] L. Kitainik, Cut technique in valued relational systems: mainsprings and applications, Fuzzy Sets and Systems 75 (1):143-164, 1995.

[18] E.P. Klement, R. Mesiar, E. Pap, Triangular Norms, Kluwer Academic Publishers, 2000.

[19] G.J. Klir, B. Yuan, Fuzzy Sets and Fuzzy Logic, Prentice Hall, 1995.

[20] J. Medina, M. Ojeda-Aciego, J. Ruiz-Calviño, On multi-adjoint concept lattices: definition and representation theorem, Lect. Notes in Artificial Intelligence 4390, pages 197-209, 2007.

[21] J. Medina, M. Ojeda-Aciego, J. Ruiz-Calviño, Formal concept analysis via multi-adjoint concept lattices, Fuzzy Sets and Systems 160 (2):130-144, 2009.

[22] Y. Djouadi, H. Prade, Interval-valued Fuzzy formal Analysis, in: Foundations of Intelligent Systems, Proceedings. Lecture Notes in Computer Sciences 5722, pages 592-601, SpringerVerlag, 2009.

[23] T.J. Ross, Fuzzy Logic With Engineering Applications, McGraw-Hill, Inc., 1995.

[24] E. Sanchez, Resolution of eigen fuzzy sets equations, Fuzzy Sets and Systems, 1 (1):69-74, 1978.

[25] M. Wagenknecht, K. Hartmenn, On the construction of fuzzy eigen solutions in given regions, Fuzzy Sets and Systems, 20 (1):55-65, 1986.

[26] R. Wille, Restructuring lattice theory: an approach based on hierarchies of concepts, in: Rival I. (Ed.), Ordered Sets, pages 445-470, Reidel, Dordrecht-Boston, 1982. 